\title{
ARTICLE
}

Epidemiology

\section{Adult height is associated with increased risk of ovarian cancer: a Mendelian randomisation study}

Suzanne C. Dixon-Suen et al. (ID

BACKGROUND: Observational studies suggest greater height is associated with increased ovarian cancer risk, but cannot exclude bias and/or confounding as explanations for this. Mendelian randomisation (MR) can provide evidence which may be less prone to bias.

METHODS: We pooled data from 39 Ovarian Cancer Association Consortium studies (16,395 cases; 23,003 controls). We applied two-stage predictor-substitution MR, using a weighted genetic risk score combining 609 single-nucleotide polymorphisms. Studyspecific odds ratios (OR) and $95 \%$ confidence intervals $(\mathrm{Cl})$ for the association between genetically predicted height and risk were pooled using random-effects meta-analysis.

RESULTS: Greater genetically predicted height was associated with increased ovarian cancer risk overall (pooled-OR (pOR) =1.06; 95\% Cl: 1.01-1.11 per $5 \mathrm{~cm}$ increase in height), and separately for invasive ( $\mathrm{pOR}=1.06 ; 95 \% \mathrm{Cl}: 1.01-1.11)$ and borderline ( $\mathrm{pOR}=$ 1.15; 95\% Cl: 1.02-1.29) tumours.

CONCLUSIONS: Women with a genetic propensity to being taller have increased risk of ovarian cancer. This suggests genes influencing height are involved in pathways promoting ovarian carcinogenesis.

British Journal of Cancer https://doi.org/10.1038/s41416-018-0011-3

\section{INTRODUCTION}

Observational studies have reported a positive association between adult height and ovarian cancer risk. ${ }^{1-4}$ However, these studies were subject to the biases inherent in conventional observational studies, including selection bias, differential and non-differential reporting bias and confounding. The degree to which these factors could account for the observed association is uncertain. Mendelian randomisation (MR) uses genetic markers as proxies for environmental exposures and, due to the singular qualities of genotype data, can provide complementary evidence by overcoming many biases affecting conventional studies. ${ }^{5}$ We used MR to examine the relationship between height and ovarian cancer risk in the Ovarian Cancer Association Consortium (OCAC), aiming to provide more certainty about the relationship between height and ovarian cancer risk. We hypothesised that greater genetically predicted height would be associated with increased risk.

\section{MATERIALS AND METHODS}

Study population and outcomes

We pooled data from 16,395 genetically European women with primary ovarian/fallopian tube/peritoneal cancer and 23,003 controls from 39 OCAC studies (Table 1; Supplementary Table 1). The data set and methods have been described previously. ${ }^{7}$ Participants were genotyped via the Collaborative Oncological Gene-Environment Study. ${ }^{8}$ Twenty-two studies provided height data (16 provided parity, oral contraceptive (OC) use, education and age at menarche information) for $>50 \%$ of their participants. We first considered all cases, then stratified by tumour behaviour. Secondary analyses stratified by histologic subtype/behaviour.

Genetic risk score

The Genetic Investigation of ANthropometric Traits (GIANT) Consortium had previously identified 697 single-nucleotide polymorphisms (SNPs) significantly associated with height. ${ }^{9}$ In our sample, 92 of these SNPs had been genotyped and the remainder were imputed using 1000 Genome Project data. ${ }^{8,} 10$ After excluding poorly-imputed SNPs (quality $\left.r^{2}<0.6\right), 609$ remained (92 genotyped/517 imputed) (Supplementary Table 2). In controls, minor allele frequencies (MAFs) were $>5 \%$ (except for 16 SNPs, MAFs 1.7-4.9\%).

We constructed a weighted genetic risk score (GRS) for height by summing height-increasing alleles across the 609 SNPs ('GRS609 '/'the GRS'), weighting alleles by $\beta$-coefficients for their association with height reported by GIANT. The score represents predicted additional height conferred by these variants, compared to having no height-increasing alleles. We report results for $5 \mathrm{~cm}$ increments.

Statistical analysis

Statistical methods have been described previously. ${ }^{7}$ Briefly, we used individual-level OCAC data for two-stage predictor-substitution $M R^{11,12}$ : first, we predicted height from the weighted GRS for 
Table 1. Characteristics of 39 OCAC studies and 39,398 participants of European ancestry included in the Mendelian randomisation analysis

\begin{tabular}{|c|c|c|c|c|c|c|c|c|}
\hline $\begin{array}{l}\text { Study } \\
\text { acronym }^{\text {a }}\end{array}$ & Country & $\begin{array}{l}\text { Diagnosis } \\
\text { (years) }\end{array}$ & $\begin{array}{l}\text { Median (range) age at } \\
\text { diagnosis/interview }\end{array}$ & $\begin{array}{l}\text { Invasive cases } \\
(N)\end{array}$ & $\begin{array}{l}\text { Borderline cases } \\
(N)\end{array}$ & $\begin{array}{l}\text { All cases } \\
(N)^{b}\end{array}$ & Controls $(N)$ & $\begin{array}{l}\text { Mean }(\mathrm{SD}) \\
\text { height }(\mathrm{cm})^{c}\end{array}$ \\
\hline AUS & Australia & 2002-2006 & $58(19-80)$ & 859 & 1 & 860 & 977 & $163(6.9)$ \\
\hline BAV & Germany & 2002-2008 & $58(24-83)$ & 96 & 5 & 102 & 143 & $164(5.8)$ \\
\hline BEL & Belgium & 2007-2010 & 46 (19-87) & 275 & 0 & 275 & 1347 & - \\
\hline DOV & USA & 2002-2009 & $57(35-74)$ & 904 & 327 & 1231 & 1487 & $166(6.5)$ \\
\hline GER & Germany & 1993-1998 & $57(21-75)$ & 189 & 24 & 213 & 413 & $163(6.0)$ \\
\hline GRR & USA & 1981-2012 & $48(21-83)$ & 125 & 0 & 125 & 0 & - \\
\hline HAW & USA & 1993-2008 & $56(27-87)$ & 60 & 20 & 80 & 157 & $163(6.6)$ \\
\hline HJO & Germany & 2007-2011 & $54(18-88)$ & 261 & 13 & 290 & 273 & - \\
\hline $\mathrm{HMO}$ & Belarus & 2006-2011 & $45(22-76)$ & 142 & 0 & 143 & 138 & - \\
\hline $\mathrm{HOC}$ & Finland & 1975-1999 & $46(18-86)$ & 210 & 8 & 239 & 447 & - \\
\hline HOP & USA & $2003-2009$ & $58(25-94)$ & 567 & 71 & 723 & 1464 & $163(6.8)$ \\
\hline HSK & Germany & 2000-2007 & $58(18-81)$ & 147 & 9 & 156 & 0 & 165 (5.6) \\
\hline LAX & USA & 1989-2008 & $58(31-88)$ & 278 & 0 & 278 & 0 & - \\
\hline MAL & Denmark & 1994-1999 & $57(31-80)$ & 440 & 138 & 578 & 828 & $166(6.1)$ \\
\hline MAY & USA & 2000-2010 & $61(20-93)$ & 699 & 79 & 778 & 743 & $165(6.3)$ \\
\hline $\mathrm{MCC}$ & Australia & 1990-2008 & $65(45-79)$ & 66 & 0 & 66 & 66 & $159(7.0)$ \\
\hline MDA & USA & 1997-2009 & $62(23-88)$ & 375 & 0 & 375 & 384 & - \\
\hline MSK & USA & 1997-2010 & $57(18-89)$ & 450 & 0 & 450 & 593 & - \\
\hline $\mathrm{NCO}$ & USA & 1999-2008 & $57(20-75)$ & 722 & 171 & 896 & 792 & $163(6.4)$ \\
\hline NEC & USA & 1992-2003 & $52(21-78)$ & 654 & 232 & 904 & 1009 & $163(6.7)$ \\
\hline NJO & USA & 2002-2009 & $60(25-88)$ & 169 & 0 & 169 & 181 & $163(6.9)$ \\
\hline NOR & Norway & 2001-2010 & $51(18-86)$ & 236 & 12 & 248 & 371 & - \\
\hline NTH & Netherlands & 1997-2008 & $55(18-83)$ & 292 & 3 & 295 & 323 & $167(6.0)$ \\
\hline ORE & USA & 2007-2011 & $58(22-86)$ & 55 & 9 & 65 & 0 & - \\
\hline OVA & Canada & $2002-2009$ & $58(19-80)$ & 640 & 161 & 801 & 748 & - \\
\hline POC & Poland & 1998-2008 & $55(23-82)$ & 423 & 0 & 423 & 417 & - \\
\hline POL & Poland & 2000-2004 & $56(24-74)$ & 236 & 0 & 236 & 223 & $162(5.6)$ \\
\hline PVD & Denmark & 2004-2009 & $63(30-88)$ & 168 & 0 & 168 & 0 & $165(6.5)$ \\
\hline $\mathrm{RMH}$ & UK & 1993-1996 & $52(26-73)$ & 148 & 7 & 155 & 0 & - \\
\hline SEA & UK & 1998-2011 & 57 (19-78) & 1447 & 76 & 1530 & 6004 & $162(6.3)$ \\
\hline SOC & UK & 1993-1998 & $62(22-92)$ & 268 & 20 & 288 & 0 & - \\
\hline SRO & UK & 1999-2001 & $59(34-84)$ & 158 & 0 & 158 & 0 & - \\
\hline STA & USA & 1997-2002 & $50(20-64)$ & 251 & 10 & 261 & 313 & $165(6.7)$ \\
\hline TOR & Canada & 1995-2007 & $58(26-85)$ & 603 & 0 & 605 & 440 & $163(7.1)$ \\
\hline $\mathrm{UCl}$ & USA & 1993-2005 & $56(18-86)$ & 277 & 141 & 418 & 367 & 165 (6.6) \\
\hline UKO & UK & $2006-2010$ & $63(19-89)$ & 718 & 0 & 718 & 1104 & $162(6.7)$ \\
\hline UKR & UK & 1991-2009 & $54(24-77)$ & 47 & 0 & 47 & 0 & - \\
\hline USC & USA & 1992-2010 & $57(22-82)$ & 693 & 152 & 845 & 1047 & $165(6.8)$ \\
\hline WOC & Poland & 1997-2010 & $44(20-81)$ & 201 & 2 & 203 & 204 & - \\
\hline
\end{tabular}

All participants were of $>90 \%$ European ancestry according to genetic markers of ancestry. ${ }^{\mathrm{a} O C A C}$ is an international collaboration of largely case-control studies. See Supplementary Table 1 for study names and references. To maximise power, nine case-only studies were grouped for analysis with case-control studies from the same region: HSK combined with GER; GRR with HOP; PVD with MAL; RMH, SOC, SRO, UKR with SEA and UKO ('UK group'); ORE with DOV; LAX with $\mathrm{UCl}$. ${ }^{\mathrm{b}}$ Cases had primary ovarian $(n=15,636)$, fallopian tube $(n=180)$ or peritoneal $(n=552)$ cancer or ovarian/tubal/peritoneal tumours of undetermined site $(n=27)$. ' Usual adult height. Height is summarised for 22 studies (20 case-control studies) where $>50 \%$ participants had data available (AUS, BAV, DOV, GER, HAW, HOP, HSK, MAL, MAY, MCC, NCO, NEC, NJO, NTH, POL, PVD, SEA, STA, TOR, UCI, UKO, USC). Sixteen of these 22 studies were also used in conventional height analyses, as they provided data on potential confounders (age, parity, use of oral contraceptives, education, and age at menarche) for $>50 \%$ of participants (AUS, DOV, GER, HAW, HOP, MAL, NCO, NEC, NJO, NTH, POL, STA, TOR, UCI, UKO, USC). OCAC Ovarian Cancer Association Consortium, SD standard deviation

all participants using coefficients from linear regression in 17,649 controls with height data; second, within each study, we used logistic regression to model disease status on GRS-predicted height. Models adjusted for age and five principal components for population structure. ${ }^{8}$ We combined study-specific estimates using meta-analysis, ${ }^{13}$ generating pooled odds ratios (pOR) and
95\% confidence intervals $(\mathrm{Cl})$ for the trend in risk per $5 \mathrm{~cm}$ increase in predicted height. We had $97 \%$ power to detect an OR of 1.10 (mRnd tool). ${ }^{14}$

Sensitivity analyses included removing 16 SNPs with MAFs $<5 \%$, and restricting to SNPs with imputation $r^{2} \geq 0.9$ ('GRS-363'), SNPs representing distinct $\operatorname{loci}^{9}$ ('GRS-377'), and directly-genotyped 


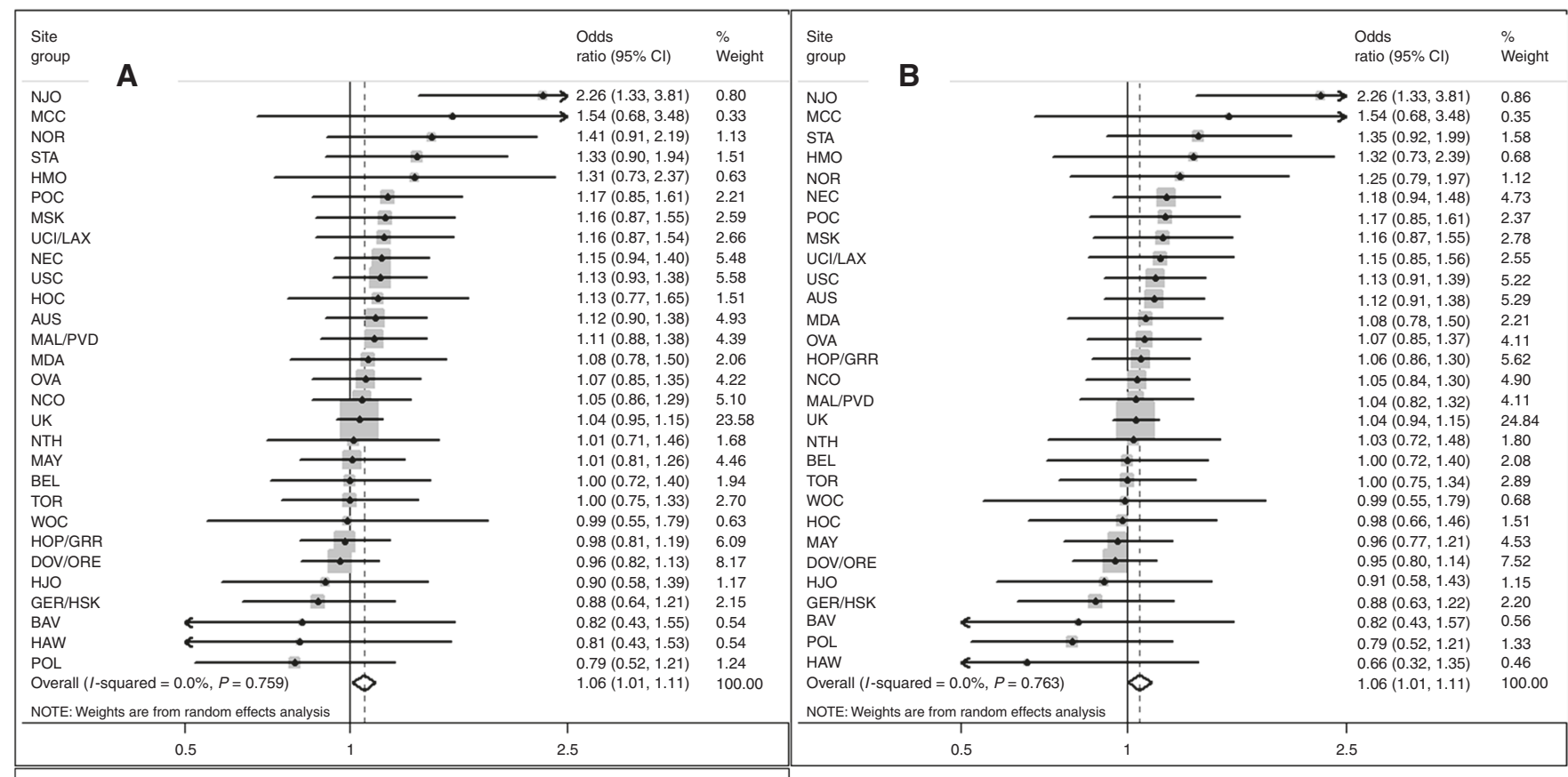

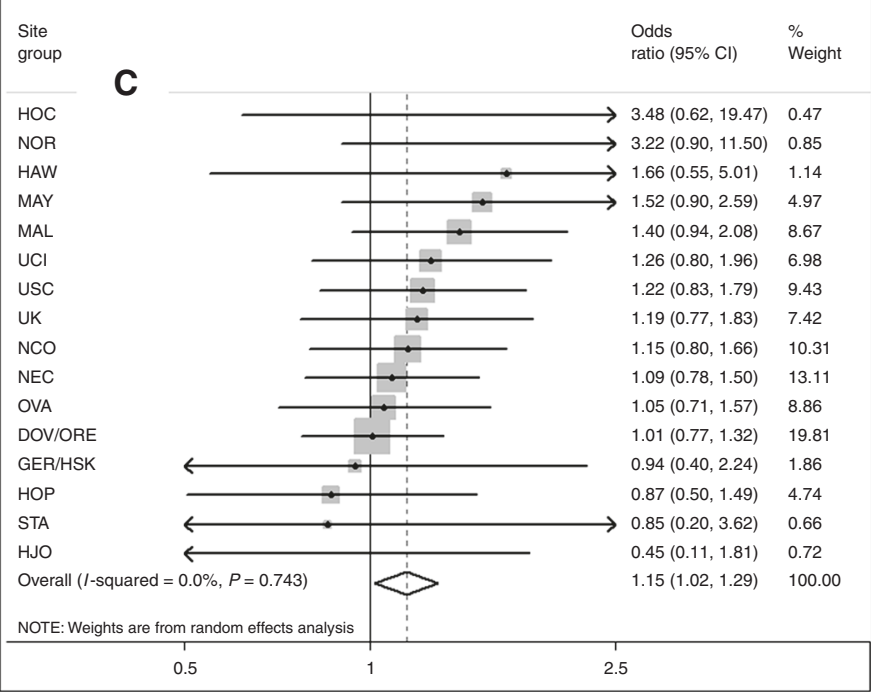

Fig. 1 Association between increasing genetically predicted height and risks of all, invasive and borderline ovarian tumours. Increasing height per $5 \mathrm{~cm}$ predicted by weighted 609-locus genetic risk score among 39 studies. Risk of a all, $\mathbf{b}$ invasive and $\mathbf{c}$ borderline ovarian tumours. The UK grouping includes RMH, SOC, SRO, UKR, SEA and UKO for $\mathbf{a}$ and $\mathbf{b}$, and RMH, SOC and SEA for $\mathbf{c}$

SNPs ('GRS-92'). We examined whether potential confounders of the association in observational studies were associated with the GRS. To assess robustness to pleiotropy (where SNPs may influence risk via pathways not mediated through height), we conducted MR-Egger regression ${ }^{15}$ and assessed smaller GRSs excluding SNPs with the highest probability of acting via other pathways from GRS to outcome (SNPs associated with ovarian/ other hormonal cancers (breast, prostate), hormone levels and in/ near tumour initiation/growth genes). We identified these potentially pleiotropic, pathway-specific SNPs via the NHGRI GWAS Catalog, ${ }^{16}$ the UCSC Genome/Table Browsers ${ }^{17,} 18$ and from lists of SNPs nominated for iCOGS genotyping by ovarian, breast and prostate cancer researchers (to capture SNPs of interest unpublished at the time of analysis).

Secondary analyses defined cases by histologic subtype/ behaviour. Among 16 studies with height/confounder data, we conducted conventional analysis (adjusted for parity, OC use, education, menarche age; stratified by study, 5-year age group) and compared results with MR-estimates among the same women.

Analyses were performed using SAS 9.2 (SAS Institute Inc., Cary, NC) and Stata 13.0 (StataCorp LP, College Station, TX). This work and each contributing study was approved by the appropriate institutional review board/ethics committee. All participants provided informed consent.

\section{RESULTS}

Population characteristics

We included 16,395 cases (14,549 invasive tumours, 1691 borderline, 155 of unknown behaviour) and 23,003 controls (Table 1). The median diagnosis year was 2003, with $74 \%$ diagnosed post- 2000 . Participants were aged 18-94 (median 56) years at diagnosis/ interview. Mean height ranged from 159 to $167 \mathrm{~cm}$ across 22 studies with data, and was 163 (standard error $(\mathrm{SE})=0.05$ ) $\mathrm{cm}$ for controls and $164(\mathrm{SE}=0.06) \mathrm{cm}$ for cases $(p<0.0001)$. 
Table 2. Association between increasing height (per $5 \mathrm{~cm}$ )—predicted by a weighted 609-locus genetic risk score-and risk of ovarian cancer, stratified by study

\begin{tabular}{|c|c|c|c|c|}
\hline Histologic subtype $^{a}$ & $N$ studies & $N$ controls & $N$ cases & Odds ratios $(95 \% \mathrm{Cl})^{\mathrm{b}}$ \\
\hline All ovarian cancers & 39 & 23,003 & 16,395 & $1.06(1.01-1.11)$ \\
\hline Borderline $^{c}$ & 20 & 16,463 & 1680 & $1.15(1.02-1.29)$ \\
\hline \multicolumn{5}{|c|}{ Secondary outcomes, by histologic subtype and behaviour } \\
\hline \multicolumn{5}{|l|}{ Serous } \\
\hline Invasive low-grade and borderline & 32 & 21,131 & 1408 & $1.15(1.01-1.30)$ \\
\hline Mucinous (invasive and borderline) & 38 & 22,410 & 1567 & $1.08(0.96-1.21)$ \\
\hline Endometrioid (invasive) & 39 & 23,003 & 2059 & $1.05(0.95-1.16)$ \\
\hline Clear cell (invasive) & 35 & 22,051 & 948 & $1.20(1.04-1.38)$ \\
\hline \multicolumn{5}{|c|}{ 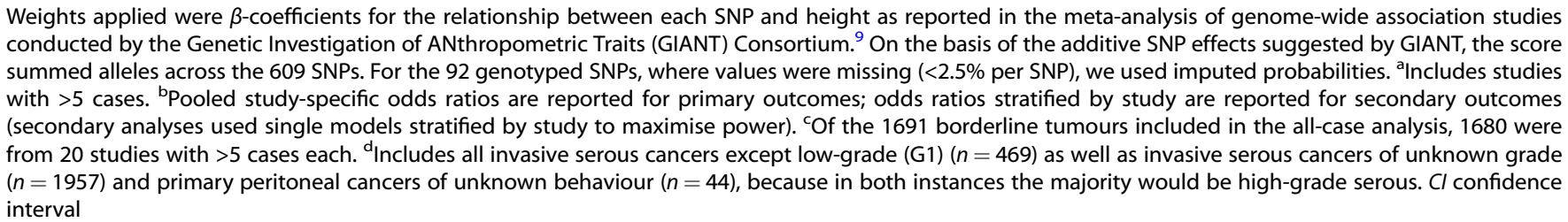 } \\
\hline
\end{tabular}

\section{Genetic risk score characteristics}

The GRS-609 was normally distributed in controls, ranging from 15.45 to 18.99 (median $=17.23$; interquartile range $=$ 16.92-17.54). It explained $13 \%$ of variance in height, $17 \%$ after adjusting for age and principal components (partial- $R^{2}=12 \%$; first-stage regression partial-F-statistic $=2505.8(\mathrm{df}=1), p<0.001)$. A 1-unit GRS-609 increase was associated with $5.2 \mathrm{~cm}$ greater height. Average height was $6.2 \mathrm{~cm}$ greater in the highest vs. lowest GRS quartile.

Cochran's $I^{2}$ and $p$-values for heterogeneity ${ }^{19}$ showed no evidence of inter-study heterogeneity in the relationship between either the GRS-609 $\left(I^{2}=34 \%, p\right.$-heterogeneity $\left.=0.07\right)$ or the simplified GRS-363 $\left(I^{2}=32 \%, p\right.$-heterogeneity $\left.=0.08\right)$ and height among controls (Supplementary Figure 1a, b). The GRS-609 was not associated with most potential confounders of the heightovarian cancer association in observational studies, including age, parity, OC use and education (Supplementary Table 3). The GRS was marginally positively associated with age at menarche $(p=$ $0.03)$, consistent with known genetic overlap between these traits. $^{20}$

\section{Primary outcomes}

Women with greater genetically predicted height had a modestly increased risk of developing ovarian cancer ( $\mathrm{pOR}=1.06,95 \% \mathrm{Cl}$ : 1.01-1.11 per $5 \mathrm{~cm}$ ) (Fig. 1a; Table 2) with a greater magnitude of association for borderline ( $\mathrm{pOR}=1.15 ; 95 \% \mathrm{Cl}$ : $1.02-1.29$ ) than invasive tumours (pOR $=1.06 ; 95 \% \mathrm{Cl}$ : 1.01-1.11; Fig. $1 \mathrm{~b}, \mathrm{c}$; Table 2). No significant inter-study heterogeneity was noted (Fig. 1a-c). GRS-363 (pOR $=1.06,95 \% \mathrm{Cl}: 1.00-1.11$, all tumours) and GRS-377 (OR $=1.07 ; 95 \% \mathrm{Cl}: 1.01-1.12)$ results were similar to the GRS-609. The association was stronger when we restricted to 92 genotyped SNPs (pOR $=1.14 ; 95 \% \mathrm{Cl}$ : 1.04-1.25). Estimates from analyses excluding low-MAF SNPs, excluding case-only studies, or adjusting for age at menarche, were similar to primary analyses. When we sequentially excluded SNPs associated with ovarian or other hormonal cancers, hormone levels and tumour development, estimates were similar or stronger than GRS-609 results. MR-Egger suggested minimal bias from pleiotropy $(p=0.1$; MR-Egger beta $=0.163$ corresponded to an OR per $5 \mathrm{~cm}$ of 1.13 (95\% Cl: 1.02-1.25), confirming a significant positive association).
In contrast, for women with height and confounder data (16 studies), the conventional analysis suggested no association (adjusted-OR $=1.01,95 \% \mathrm{Cl}: 0.99-1.04$ per $5 \mathrm{~cm}$ ). Conducting MR within the same 16 studies yielded results similar to overall analyses (OR $=1.06,95 \% \mathrm{Cl}: 1.00-1.13)$ (Supplementary Table 4).

\section{Secondary outcomes}

After stratifying by subtype/behaviour, the strongest associations were seen for clear cell $(\mathrm{OR}=1.20,95 \% \mathrm{Cl}: 1.04-1.38)$ and lowgrade/borderline serous cancers $(\mathrm{OR}=1.15,95 \% \mathrm{Cl}: 1.01-1.30)$ (Table 2). However, Cls were wide and overlapping due to lower statistical power in these subgroup analyses. The estimate for clear cell cancers was also significantly elevated in our conventional analyses (Supplementary Table 4).

\section{DISCUSSION}

We used a 609-SNP GRS to examine the relationship between height and ovarian cancer risk for women of European ancestry. Our data indicate a modest positive association between genetically predicted height and ovarian cancer risk, which may be stronger for borderline cancers. Height may be relevant to cancer risk as a marker for lifetime growth-factor levels (e.g. IGF-1) and/or early-life exposures (socio-economic/environmental/nutritional). $3,21,22$

Observational studies are subject to biases (reverse causality, selection bias, differential/non-differential reporting, confounding) which cannot be ruled out as possible explanations for observed associations. By using genotype, the MR technique can overcome some of these biases, given three assumptions. We confirmed the two verifiable assumptions: the GRS was associated with height, and not with most known confounders. The GRS-menarche age association is unlikely to explain the observed association, because age at menarche is only weakly associated with ovarian cancer, and women with later menarche have if anything lower ovarian cancer risk, so if this affected our results, we would expect the true effect to be at least as strong as the reported association. Also, removing hormone-related SNPs, or adjusting for menarche age, did not attenuate estimates. Owing to the limited current biological understanding of all 609 SNPs, we could not 
conclusively exclude the presence of alternate pathways from height genes to ovarian cancer (assumption three). However, MREgger and sensitivity analyses excluding pathway-specific SNPs provided some evidence for their absence, minimising the likelihood that our observed association is explained by pathways separate from height/growth. Although height data were not available for the entire population, this is unlikely to have affected our results as we used these data only to refine the height predictions from the GRS, and there is no reason to believe the GRS-height relationship would be different for women with and without height data. Further strengths of our analysis include the large number of SNPs and power to detect modest differences.

Despite potential limitations of conventional observational studies, our MR-estimate is almost identical to previously reported associations, suggesting previous estimates were not appreciably biased. The World Cancer Research Fund/American Institute for Cancer Research meta-analysis of 24 prospective studies, and a study pooling 47 prospective/case-control studies, both reported a significant $7-8 \%$ increase in risk (combining invasive/borderline cancers) per $5 \mathrm{~cm}$ height increase. ${ }^{3,4}$ The lack of association seen in the OCAC conventional height analysis reflects the greater potential for bias in case-control studies and illustrates the value of $\mathrm{MR}$ in overcoming these biases. Few previous studies have examined borderline cancers separately, a strength of our analysis. Previous observational studies have not reported consistent patterns by histologic subtype ${ }^{2,4}$, 23; our secondary analyses were under-powered to resolve this question.

Using MR, we have established that the previously observed association between height and ovarian cancer risk is unlikely to have been explained by bias, and that genetic factors influencing height play roles in ovarian cancer development. Height could therefore be used, with other risk factors, to identify women at elevated risk. Further research should continue to explore mechanisms underpinning this association.

\section{ACKNOWLEDGEMENTS}

We thank all the individuals who took part in this study and all the researchers, clinicians, and technical and administrative staff who have made possible the many studies contributing to this work. In particular, for their contribution to the design and conduct of the individual studies that contributed to the analysis, we thank: D. Bowtell, A. deFazio, D. Gertig, A. Green, P. Parsons, N. Hayward and D. Whiteman (AUS); G. Peuteman, T. Van Brussel and D. Smeets (BEL); U. Eilber (GER); S. Reckemeyer, A. Korotkaia and S. Reina-Campanon (HJO); J. Meyer, C. Hilker, S. Windebank, and J. Vollenweider (MAY); the Victorian Cancer Registry and the Australian Institute of Health and Welfare for ascertaining cases and vital status (MCC); I. Orlow, L. Paddock, and L. Rodriguez-Rodriguez (NJO); the SEARCH team, C. Luccarini, C. Baynes, and D. Conroy (SEA); the Scottish Gynaecological Clinical Trials group and SCOTROC1 investigators (SRO); I. Jacobs, M.Widschwendter, E. Wozniak, A Ryan, J. Ford, N. Balogun and C. Karpinskyj (UKO); and C. Pye (UKR).

\section{ADDITIONAL INFORMATION}

Supplementary information is available for this paper at https://doi.org/10.1038/ s41416-018-0011-3.

\section{Competing interests: The authors declare no competing financial interests.}

Funding: This work was supported by: the National Cancer Institute at the U.S. National Institutes of Health [K07-CA095666, K07-CA80668, K07-CA143047, K22CA138563, N01-CN025403, N01-CN55424, N01-PC67001, N01-PC67010, P01-CA17054, P30-CA072720, P30-CA008748, P30-CA14089, P30-CA15083, P50-CA105009, P50CA136393, P50-CA159981, R01-CA058860, R01 CA063678, R01 CA063682, R01CA092044, R01-CA095023， R01-CA16056, R01-CA54419, R01-CA58598, R01CA61107, R01-CA61132, R01-CA76016, R01-CA83918, R01-CA87538, R01-CA112523, R01-CA122443, R03-CA113148, R03-CA115195, U01-CA69417, U01-CA71966 and Intramural Research funds]; the European Commission's Seventh Framework Programme [agreement number 223175 HEALTH F2 2009-223175]; Cancer Research UK [C490/A16561, C536/A13086, C536/A6689, C1287/A10118, C1287/A 10710, C12292/A11174, C1281/A12014, C5047/A8384, C5047/A15007, C5047/A10692 and C8197/A16565]; a National Institutes of Health (CA128978), Cancer Post-GWAS
Initiative [1U19 CA148537, 1U19 CA148065 and 1U19 CA148112-the Genetic Associations and Mechanisms in Oncology (GAME-ON) initiative]; the U.S. Department of Defense [DAMD17-02-1-0669, W81XWH-07-0449, DAMD17-02-1-0666, W81XWH-10-1-0280 and W81XWH-10-1-0341]; the Canadian Institutes of Health Research (CIHR) [MOP-86727 and MSH-87734 to L.E.K.] and the CIHR Team in Familial Risks of Breast Cancer; the Komen Foundation for the Cure; the Breast Cancer Research Foundation; the Ovarian Cancer Research Fund (thanks to donations by the family and friends of Kathryn Sladek Smith); the U.S. Army Medical Research and Materiel Command [DAMD17-01-1-0729 and DAMD17-02-1-0669]; the National Health and Medical Research Council of Australia [199600, 209057, 251553, 400281, 504711, 1073898 and fellowships to G.C.-T. and P.M.W.]; Cancer Councils of Queensland, Victoria, New South Wales, South Australia and Tasmania and the Cancer Foundation of Western Australia [Multi-State Grant Applications 191, 211 and 182]; VicHealth; the ELAN Program of the University of Erlangen-Nuremberg; the Nationaal Kankerplan of Belgium; the German Federal Ministry of Education and Research of Germany Programme of Clinical Biomedical Research [01 GB 9401]; the German Cancer Research Center; the Roswell Park Cancer Institute Alliance Foundation [P30 CA016056]; the Rudolf-Bartling Foundation; the Helsinki University Central Hospital Research Fund; the National Institutes of Health/National Center for Research Resources/General Clinical Research Center [M01-RR000056]; an American Cancer Society Early Detection Professorship [SIOP-06-258-01-COUN to B.Y.K.]; the National Center for Advancing Translational Sciences (NCATS) [UL1TR000124 to B.Y. K.]; the Danish Cancer Society [94-222-52]; the Mermaid I project; the Mayo Foundation; the Minnesota Ovarian Cancer Alliance; the Fred C. and Katherine B. Andersen Foundation; the Cancer Institute of New Jersey; Helse Vest; the Norwegian Cancer Society; the Research Council of Norway; Radboud University Medical Centre; the Oregon Health and Science University (OHSU) Foundation; Pomeranian Medical University; the UK National Institute for Health Research Biomedical Research Centres at the University of Cambridge, University College London Hospital, and the Royal Marsden Hospital; the Imperial Experimental Cancer Research Centre [C1312/ A15589]; the U.S. Public Health Service [PSA-042205]; the Lon V. Smith Foundation [LVS-39420]; The Eve Appeal; The Oak Foundation; the California Cancer Research Program [00-01389V-20170 and 2ll0200]; the Polish Ministry of Science and Higher Education [4 PO5C 02814 and 2 PO5A 068 27]; and the Maria Sklodowska-Curie Memorial Cancer Center and Institute of Oncology, Warsaw.

Note: This work is published under the standard license to publish agreement. After 12 months the work will become freely available and the license terms will switch to a Creative Commons Attribution 4.0 International licence (CC BY 4.0).

\section{REFERENCES}

1. Emerging Risk Factors Collaboration. Adult height and the risk of cause-specific death and vascular morbidity in 1 million people: individual participant metaanalysis. Int. J. Epidemiol. 41, 1419-1433 (2012).

2. Schouten, L. J. et al. Height, body mass index, and ovarian cancer: a pooled analysis of 12 cohort studies. Cancer Epidemiol. Biomark. Prev. 17, 902-912 (2008).

3. World Cancer Research Fund/American Institute for Cancer Research. Food, Nutrition, Physical Activity, and the Prevention of Ovarian Cancer 2014 (AICR, Washington DC, 2014).

4. Collaborative Group on Epidemiological Studies of Ovarian Cancer. Ovarian cancer and body size: individual participant meta-analysis including 25,157 women with ovarian cancer from 47 epidemiological studies. PLoS Med. 9, e1001200 (2012).

5. Davey Smith, G. \& Ebrahim, S. 'Mendelian randomization': can genetic epidemiology contribute to understanding environmental determinants of disease? Int. J. Epidemiol. 32, 1-22 (2003).

6. Gayther, S. A. et al. Tagging single nucleotide polymorphisms in cell cycle control genes and susceptibility to invasive epithelial ovarian cancer. Cancer Res. 67, 3027-3035 (2007).

7. Dixon, S. C. et al. Adult body mass index and risk of ovarian cancer by subtype: a Mendelian randomization study. Int. J. Epidemiol. 45, 884-895 (2016).

8. Pharoah, P. D. P. et al. GWAS meta-analysis and replication identifies three new susceptibility loci for ovarian cancer. Nat. Genet. 45, 362-370 (2013).

9. Wood, A. R. et al. Defining the role of common variation in the genomic and biological architecture of adult human height. Nat. Genet. 46, 1173-1186 (2014).

10. Abecasis, G. R. et al. A map of human genome variation from population-scale sequencing. Nature 467, 1061-1073 (2010).

11. Burgess, S. Identifying the odds ratio estimated by a two-stage instrumental variable analysis with a logistic regression model. Stat. Med. 32, 4726-4747 (2013).

12. Didelez, V., Meng, S. \& Sheehan, N. A. Assumptions of IV methods for observational epidemiology. Stat. Sci. 25, 22-40 (2010). 
13. Stukel, T. A., Demidenko, E., Dykes, J. \& Karagas, M. R. Two-stage methods for the analysis of pooled data. Stat. Med. 20, 2115-2130 (2001).

14. Brion, M. J., Shakhbazov, K. \& Visscher, P. M. Calculating statistical power in Mendelian randomization studies. Int. J. Epidemiol. 42, 1497-1501 (2013).

15. Bowden, J., Davey Smith, G. \& Burgess, S. Mendelian randomization with invalid instruments: effect estimation and bias detection through Egger regression. Int. J. Epidemiol. 44, 512-525 (2015).

16. Welter, D. et al. The NHGRI GWAS Catalog, a curated resource of SNP-trait associations. Nucleic Acids Res. 42, D1001-D1006 (2014).

17. Karolchik, D. et al. The UCSC Table Browser data retrieval tool. Nucleic Acids Res. 32, D493-D496 (2004).

18. Kent, W. J. et al. The human genome browser at UCSC. Genome Res. 12, 996-1006 (2002).
19. Higgins, J. P. T. \& Thompson, S. G. Quantifying heterogeneity in a meta-analysis. Stat. Med. 21, 1539-1558 (2002).

20. Bulik-Sullivan, B. et al. An atlas of genetic correlations across human diseases and traits. Nat. Genet. 47, 1236-1241 (2015).

21. Stefan, N., Haring, H. U., Hu, F. B. \& Schulze, M. B. Divergent associations of height with cardiometabolic disease and cancer: epidemiology, pathophysiology, and global implications. Lancet Diabetes Endocrinol. 4, 457-467 (2016).

22. Clayton, P., Banerjee, I., Renehan, A. G., Murray, P. G. \& Clayton, P. E. Growth hormone, the insulin-like growth factor axis, insulin and cancer risk. Nat. Rev. Endocrinol. 7, 11-24 (2011).

23. Jordan, S. J., Webb, P. M. \& Green, A. C. Height, age at menarche, and risk of epithelial ovarian cancer. Cancer Epidemiol. Biomark. Prev. 14, 2045-2048 (2005).

\section{AFFILIATIONS}

Suzanne C. Dixon-Suen (D) ${ }^{1,2}$, Christina M. Nagle ${ }^{1,2}$, Aaron P. Thrift ${ }^{3}$, Paul D. P. Pharoah ${ }^{4}$, Ailith Ewing ${ }^{4}$, Celeste Leigh Pearce ${ }^{5,6}$, Wei Zheng ${ }^{7}{ }^{7}$, Australian Ovarian Cancer Study Group ${ }^{1,8}$, Georgia Chenevix-Trench ${ }^{9}$, Peter A. Fasching ${ }^{10,11}$, Matthias W. Beckmann ${ }^{11}$, Diether Lambrechts ${ }^{12,13}$, Ignace Vergote ${ }^{14}$, Sandrina Lambrechts ${ }^{14}$, Els Van Nieuwenhuysen ${ }^{14}$, Mary Anne Rossing ${ }^{15,16}$, Jennifer A. Doherty ${ }^{17}$, Kristine G. Wicklund ${ }^{15}$, Jenny Chang-Claude ${ }^{18,19}$, Audrey Y. Jung ${ }^{18}$, Kirsten B. Moysich ${ }^{20}$, Kunle Odunsi ${ }^{21}$, Marc T. Goodman 22,23, Lynne R. Wilkens ${ }^{24}$, Pamela J. Thompson ${ }^{22}$, Yurii B. Shvetsov ${ }^{24}$, Thilo Dörk ${ }^{25}$, Tjoung-Won Park-Simon ${ }^{25}$, Peter Hillemanns ${ }^{25}$, Natalia Bogdanova ${ }^{26}$, Ralf Butzow ${ }^{27}$, Heli Nevanlinna ${ }^{28}$, Liisa M. Pelttari ${ }^{28}$, Arto Leminen ${ }^{28}$, Francesmary Modugno ${ }^{29,30,31}$, Roberta B. Ness ${ }^{32}$, Robert P. Edwards ${ }^{29,30}$, Joseph L. Kelley ${ }^{29}$, Florian Heitz ${ }^{33,34}$, Andreas du Bois ${ }^{33,34}$, Philipp Harter ${ }^{33,34}$, Ira Schwaab ${ }^{35}$, Beth Y. Karlan ${ }^{36}$, Jenny Lester ${ }^{36}$, Sandra Orsulic ${ }^{36}$, Bobbie J. Rimel ${ }^{36}$, Susanne K. Kjær ${ }^{37,38}$, Estrid Høgdall $^{37,39}$, Allan Jensen ${ }^{37}$, Ellen L. Goode ${ }^{40}$, Brooke L. Fridley ${ }^{41}$, Julie M. Cunningham ${ }^{42}$, Stacey J. Winham ${ }^{43}$, Graham G. Giles ${ }^{44,45,46}$, Fiona Bruinsma ${ }^{44}$, Roger L. Milne ${ }^{44,45}$, Melissa C. Southey ${ }^{47}$, Michelle A. T. Hildebrandt ${ }^{48}, \mathrm{Xifeng}^{\prime} \mathrm{Wu}^{48}$, Karen H. Lu ${ }^{49}$, Dong Liang ${ }^{50}$, Douglas A. Levine ${ }^{51}$, Maria Bisogna ${ }^{52}$, Joellen M. Schildkraut ${ }^{53}$, Andrew Berchuck ${ }^{54}$, Daniel W. Cramer ${ }^{55}$, Kathryn L. Terry ${ }^{55,56}$, Elisa V. Bandera ${ }^{57,58}$, Sara H. Olson ${ }^{59}$, Helga B. Salvesen ${ }^{60,61}$, Liv Cecilie Vestrheim Thomsen ${ }^{60,61}$, Reidun K. Kopperud ${ }^{60,61}$, Line Bjorge ${ }^{60,61}$, Lambertus A. Kiemeney ${ }^{62}$, Leon F. A. G. Massuger ${ }^{63}$, Tanja Pejovic ${ }^{64,65}$, Amanda Bruegl ${ }^{64}$, Linda S. Cook ${ }^{66}$, Nhu D. Le ${ }^{67}$, Kenneth D. Swenerton ${ }^{68}$, Angela Brooks-Wilson ${ }^{69,70}$, Linda E. Kelemen ${ }^{71}$, Jan Lubiński ${ }^{72}$, Tomasz Huzarski $^{72}$, Jacek Gronwald ${ }^{72}$, Janusz Menkiszak ${ }^{73}$, Nicolas Wentzensen ${ }^{74}$, Louise Brinton (D) $^{74}$, Hannah Yang ${ }^{74}$, Jolanta Lissowska ${ }^{75}$, Claus K. Høgdall ${ }^{38}$, Lene Lundvall ${ }^{38}$, Honglin Song ${ }^{4}$, Jonathan P. Tyrer ${ }^{4}$, lan Campbell ${ }^{76,77}$, Diana Eccles ${ }^{78}$, James Paul ${ }^{79}$, Rosalind Glasspool (iD ${ }^{80}$, Nadeem Siddiqui ${ }^{81}$, Alice S. Whittemore ${ }^{82}$, Weiva Sieh ${ }^{83}$, Valerie McGuire ${ }^{82}$, Joseph H. Rothstein ${ }^{83}$, Steven A. Narod ${ }^{84}$, Catherine Phelan ${ }^{85}$, Harvey A. Risch ${ }^{86}$, John R. McLaughlin ${ }^{87}$, Hoda Anton-Culver ${ }^{88,89}$, Argyrios Ziogas ${ }^{88}$, Usha Menon ${ }^{90}$, Simon A. Gayther ${ }^{91}$, Susan J. Ramus ${ }^{92,93}$, Aleksandra Gentry-Maharaj ${ }^{90}$, Anna H. Wu $^{6}$, Malcolm C. Pike ${ }^{6,59}$, Chiu-Chen Tseng ${ }^{6}$, Jolanta Kupryjanczyk ${ }^{94}$, Agnieszka Dansonka-Mieszkowska94, Agnieszka Budzilowska ${ }^{94}$, Iwona K. Rzepecka ${ }^{94}$ and Penelope M. Webb ${ }^{1,2}$, on behalf of the Ovarian Cancer Association Consortium

${ }^{1}$ Gynaecological Cancers Group, QIMR Berghofer Medical Research Institute, 300 Herston Road, Brisbane QLD 4006, Australia; ${ }^{2}$ The University of Queensland, School of Public Health, Level 2 Public Health Building (887), Corner of Herston Road \& Wyndham Street, Brisbane QLD 4006, Australia; ${ }^{3}$ Department of Medicine and Dan L Duncan Comprehensive Cancer Center, Baylor College of Medicine, One Baylor Plaza, Houston, TX 77030, USA; ${ }^{4}$ Strangeways Research Laboratory, Centre for Cancer Genetic Epidemiology, Department of Public Health \& Primary Care/Department of Oncology, University of Cambridge, Worts Causeway, Cambridge CB1 8RN, UK; ${ }^{5}$ Department of Epidemiology, University of Michigan School of Public Health, 1415 Washington Heights, SPH Tower, Ann Arbor, MI 48109-2029, USA; ${ }^{6}$ Department of Preventive Medicine, Keck School of Medicine, University of Southern California Norris Comprehensive Cancer Center, 1441 Eastlake Avenue, Los Angeles, CA 90033, USA; ${ }^{7}$ Vanderbilt Epidemiology Center, Vanderbilt University School of Medicine, 2525 West End Ave., Nashville, TN 37203, USA; ${ }^{8}$ Cancer Genetics and Genomics Laboratory, Peter MacCallum Cancer Centre, St Andrews Place, Melbourne, VIC 3002, Australia; ${ }^{9}$ Cancer Genetics Group, QIMR Berghofer Medical Research Institute, 300 Herston Road, Brisbane QLD 4006, Australia; ${ }^{10}$ Department of Medicine, Division of Hematology and Oncology, David Geffen School of Medicine, University of California at Los Angeles, 10833 Le Conte Ave, Los Angeles, CA 90095, USA; ${ }^{11}$ Department of Gynecology and Obstetrics, University Hospital Erlangen, Friedrich-Alexander-University Erlangen-Nuremberg, Comprehensive Cancer Center Erlangen-EMN, Universitätsstrasse 21-23, 91054 Erlangen, Germany; ${ }^{12}$ Vesalius Research Center, VIB, Herestraat 49, bus 912,3000 Leuven, Belgium; ${ }^{13}$ Laboratory for Translational Genetics, Department of Oncology, University of Leuven, O\&N IV Herestraat 49-Box 912, 3000 Leuven, Belgium; ${ }^{14}$ Division of Gynecologic Oncology, Department of Obstetrics and Gynaecology and Leuven Cancer Institute, University Hospitals Leuven, Herestraat 49, Leuven 3000, Belgium; ${ }^{15}$ Program in Epidemiology, Division of Public Health Sciences, Fred Hutchinson Cancer Research Center, 1100 Fairview Ave. N., Seattle, WA 98109-1024, USA; ${ }^{16}$ Department of Epidemiology, University of Washington, 1959 NE Pacific Street, Health Sciences Bldg, F262, Seattle, WA 98195, USA; ${ }^{17}$ Department of Epidemiology, The Geisel School of Medicine at Dartmouth, 1 Medical Center Drive, 7927 Rubin Building, Lebanon, NH 03756, USA; ${ }^{18}$ Division of Cancer Epidemiology, German Cancer Research Center, Im Neuenheimer Feld 581, Heidelberg 69120, Germany; ${ }^{19}$ University Cancer Center Hamburg, University Medical Center Hamburg-Eppendorf, Martinistr. 52, 20246 Hamburg, Germany; ${ }^{20}$ Department of Cancer Prevention and Control, Roswell Park Cancer Institute, Elm and Carlton Streets, Buffalo, NY 14263, USA; ${ }^{21}$ Department of Gynecological Oncology, Roswell Park Cancer Institute, Elm and Carlton Streets, Buffalo, NY 14263, USA; ${ }^{22}$ Cancer Prevention and Control, Samuel Oschin Comprehensive Cancer Institute, Cedars-Sinai Medical Center, 8700 Beverly Blvd., Los Angeles, CA 90048, USA; ${ }^{23}$ Community and Population Health Research Institute, Department of Biomedical Sciences, Cedars-Sinai Medical Center, 8700 Beverly Blvd., Los Angeles, CA 90048 , USA; ${ }^{24}$ Cancer Epidemiology Program, University of Hawaii Cancer Center, 701 llalo Street, Honolulu, HI 96813, USA; ${ }^{25}$ Clinics of Obstetrics and Gynaecology, Hannover Medical School, Carl-Neuberg-Str. 1, D-30625 Hannover, Germany; ${ }^{26}$ Radiation Oncology Research Unit, Hannover Medical School, Carl-Neuberg-Str. 1, D-30625 Hannover, Germany; ${ }^{27}$ Department of Pathology, University of Helsinki and Helsinki University Hospital, 
Haartmaninkatu 8, 00029 Helsinki, Finland; ${ }^{28}$ Department of Obstetrics and Gynecology, University of Helsinki and Helsinki University Hospital, Haartmaninkatu 8, 00029 Helsinki, Finland; ${ }^{29}$ Division of Gynecologic Oncology, Department of Obstetrics, Gynecology and Reproductive Sciences, University of Pittsburgh School of Medicine, 300 Halket Street, Pittsburgh, PA 15213, USA; ${ }^{30}$ Ovarian Cancer Center of Excellence, Women's Cancer Research Program, Magee-Women's Research Institute and University of Pittsburgh Cancer Institute, 204 Craft Avenue, Pittsburgh, PA 15213, USA; ${ }^{31}$ Department of Epidemiology, University of Pittsburgh Graduate School of Public Health, 130 De Soto Street, Pittsburgh, PA 15261, USA; ${ }^{32}$ The University of Texas Health Science Center at Houston, School of Public Health, 1200 Herman Pressler, Suite E-1015, Houston, TX 77030, USA; ${ }^{33}$ Department of Gynecology and Gynecologic Oncology, Kliniken Essen-Mitte/ Evang. Huyssens-Stiftung/ Knappschaft GmbH, Henricistrasse 92, 45136 Essen, Germany; ${ }^{34}$ Department of Gynecology and Gynecologic Oncology, Dr. Horst Schmidt Kliniken Wiesbaden, Ludwig-Erhard-Strasse 100, 65199 Wiesbaden, Germany; ${ }^{35}$ Praxis für Humangenetik, Biebricher Allee 117, 65187 Wiesbaden, Germany; ${ }^{36}$ Women's Cancer Program at the Samuel Oschin Comprehensive Cancer Institute, Cedars-Sinai Medical Center, 8635 West Third Street, Los Angeles, CA 90048, USA; ${ }^{37}$ Department of Virus, Lifestyle and Genes, Danish Cancer Society Research Center, Strandboulevarden 49, DK-2100 Copenhagen, Denmark; ${ }^{38}$ Department of Gynaecology, Rigshospitalet, University of Copenhagen, Blegdamsvej 9, DK-2100 Copenhagen, Denmark; ${ }^{39}$ Molecular Unit, Department of Pathology, Herlev Hospital, University of Copenhagen, Herlev Ringvej 75, DK-2370 Herlev, Denmark; ${ }^{40}$ Department of Health Science Research, Division of Epidemiology, Mayo Clinic, 200 First Street SW, Charlton 6, Rochester, MN 55905, USA; ${ }^{41}$ Department of Biostatistics and Bioinformatics, Moffitt Cancer Center, 12902 Magnolia Drive, Tampa, FL 33612, USA; ${ }^{42}$ Department of Laboratory Medicine and Pathology, Mayo Clinic, 200 First Street SW, Stabile 13, Rochester, MN 55905, USA; ${ }^{43}$ Division of Biomedical Statistics and Informatics, Department of Health Science Research, Mayo Clinic, 200 First Street SW, Charlton 6, Rochester, MN 55905, USA; ${ }^{44}$ Cancer Epidemiology and Intelligence Division, Cancer Council Victoria, 615 St Kilda Road, Melbourne VIC 3004, Australia; ${ }^{45}$ Centre for Epidemiology and Biostatistics, Melbourne School of Population and Global Health, The University of Melbourne, Grattan Street, Parkville VIC 3010, Australia; ${ }^{46}$ Department of Epidemiology and Preventive Medicine, Monash University, The Alfred Centre, 99 Commercial Road, Melbourne VIC 3004, Australia; ${ }^{47}$ Genetic Epidemiology Laboratory, Department of Pathology, The University of Melbourne, Grattan Street, Carlton VIC 3053, Australia; ${ }^{48}$ Department of Epidemiology, The University of Texas MD Anderson Cancer Center, 1155 Pressler Blvd-Unit 1340, Houston, TX 77030, USA; ${ }^{49}$ Department of Gynecologic Oncology, The University of Texas MD Anderson Cancer Center, 1155 Pressler Blvd - Unit 1340, Houston, TX 77030, USA; ${ }^{50}$ College of Pharmacy and Health Sciences, Texas Southern University, 3100 Cleburne St, Houston, TX 77004, USA; ${ }^{51}$ Division of Gynecologic Oncology, Department of Obstetrics And Gynecology, NYU Langone Medical Center, 240 East 38th Street, New York, NY 10016, USA; ${ }^{52}$ Gynecology Service, Department of Surgery, Memorial Sloan Kettering Cancer Center, 417 East 68th Street, New York, NY 10065, USA; ${ }^{53}$ Department of Public Health Sciences, The University of Virginia, Box 800717, Charlotteville, VA 22908, USA; ${ }^{54}$ Department of Obstetrics and Gynecology, Duke University Medical Center, 25171 Morris Bldg, Durham, NC 27710, USA;

${ }^{55}$ Obstetrics and Gynecology Epidemiology Center, Brigham and Women's Hospital, 221 Longwood Avenue, Richardson Fuller Building, Boston, MA 02115, USA; ${ }^{56}$ Department of Epidemiology, Harvard T.H. Chan School of Public Health, 677 Huntington Ave, Boston, MA 02115, USA; ${ }^{57}$ Cancer Prevention and Control Program, Rutgers Cancer Institute of New Jersey, 195 Little Albany Street, New Brunswick, NJ 08903, USA; ${ }^{58}$ Rutgers School of Public Health, 683 Hoes Lane West, Piscataway, NJ 08854, USA; ${ }^{59}$ Department of Epidemiology and Biostatistics, Memorial Sloan Kettering Cancer Center, 485 Lexington Ave, New York, NY 10017, USA; ${ }^{60}$ Department of Obstetrics and Gynecology, Haukeland University Hospital, Kvinneklinikken, Jonas Liesvei 72, 5058 Bergen, Norway; ${ }^{61}$ Centre for Cancer Biomarkers, Department of Clinical Science, University of Bergen, Postboks 7804, N-5020 Bergen, Norway; ${ }^{62}$ Radboud University Medical Center, Radboud Institute for Health Sciences, PO Box 9101,6500 HB Nijmegen, The Netherlands; ${ }^{63}$ Radboud University Medical Center, Radboud Institute for Molecular Life Sciences, Department of Gynaecology, PO Box 9101, 6500 HB Nijmegen, The Netherlands; ${ }^{64}$ Department of Obstetrics \& Gynecology, Oregon Health \& Science University, 3181 SW Sam Jackson Park Road, Portland, OR 97239, USA; ${ }^{65}$ Knight Cancer Institute, Oregon Health \& Science University, 3181 SW Sam Jackson Park Road, Portland, OR 97239, USA; ${ }^{6}$ Division of Epidemiology and Biostatistics, Department of Internal Medicine, University of New Mexico, 2703 Frontier Ave NE, Albuquerque, NM 87131, USA; ${ }^{67}$ Cancer Control Research, BC Cancer Agency, 675 West 10th Avenue, Vancouver, BC, Canada;

${ }^{68}$ Department of Medicine, University of British Columbia, 2775 Laurel Street, Vancouver, BC V5Z 1M9, Canada; ${ }^{69}$ Canada's Michael Smith Genome Sciences Centre, BC Cancer Agency, 675 West 10th Avenue, Vancouver, BC, Canada; ${ }^{70}$ Department of Biomedical Physiology and Kinesiology, Simon Fraser University, 8888 University Drive, Burnaby, BC V5A 1S6, Canada; ${ }^{71}$ Department of Public Health Sciences, Medical University of South Carolina, 68 President Street, Bioengineering Building, Charleston, SC 29425, USA; ${ }^{2}$ International Hereditary Cancer Center, Department of Genetics and Pathology, Pomeranian Medical University, ul. Połabska 4, Szczecin 70-115, Poland; ${ }^{73}$ Department of Gynecological Surgery and Gynecological Oncology of Adults and Adolescents, Pomeranian Medical University, ul. Powstańców Wlkp 72, 70-111 Szczecin, Poland; ${ }^{74}$ Division of Cancer Epidemiology and Genetics, National Cancer Institute, 9609 Medical Center Drive, Rockville, MD 20850, USA; ${ }^{75}$ Department of Cancer Epidemiology and Prevention, The Maria Sklodowska-Curie Memorial Cancer Center and Institute of Oncology, Wawelska 15B, 02-034 Warsaw, Poland; ${ }^{76}$ Cancer Genetics Laboratory, Research Division, Peter MacCallum Cancer Centre, St Andrews Place, Melbourne VIC 3002, Australia; ${ }^{77}$ Department of Pathology, University of Melbourne, Grattan Street, Carlton, VIC 3053, Australia; ${ }^{78}$ Faculty of Medicine, Southampton University Hospitals Trust, Princess Anne Hospital, University of Southampton, Southampton SO16 5YA, UK; ${ }^{79}$ Cancer Research UK Clinical Trials Unit Glasgow, Institute of Cancer Sciences, University of Glasgow, $1053 \mathrm{Gt}$. Western Road, Glasgow G12 0YN, UK; ${ }^{80}$ The Beatson West of Scotland Cancer Centre, 1053 Gt. Western Road, Glasgow G12 OYN, UK; ${ }^{81}$ Department of Gynaecological Oncology, Glasgow Royal Infirmary, Alexandra Parade, Glasgow G31 2ER, UK; ${ }^{82}$ Department of Health Research and Policy-Epidemiology, Stanford University School of Medicine, HRP Redwood Building, 259 Campus Drive, Stanford, CA 94305, USA; ${ }^{83}$ Departments of Population Health Science \& Policy and Genetics \& Genomic Sciences, Icahn School of Medicine at Mount Sinai, 1 Gustave L. Levy Place, New York, NY 10029, USA; ${ }^{84}$ Women's College Research Institute, University of Toronto, 790 Bay Street, Toronto, ON M5G 1N8, Canada; ${ }^{85}$ Department of Cancer Epidemiology, Moffitt Cancer Center, 12902 Magnolia Drive, Tampa, FL 33612, USA; ${ }^{86}$ Department of Chronic Disease Epidemiology, Yale School of Public Health, LEPH 413, 60 College Street, New Haven, CT 06510, USA; ${ }^{87}$ Public Health Ontario, 480 University Avenue (/300), Toronto, ON M5G1V2, Canada; ${ }^{88}$ Department of Epidemiology, University of California Irvine, 224 Irvine Hall, Irvine, CA 92697-7550, USA; ${ }^{89}$ Genetic Epidemiology Research Institute, UCI Center for Cancer Genetics Research \& Prevention, School of Medicine, University of California Irvine, 224 Irvine Hall, Irvine, CA 92697-7550, USA; ${ }^{90}$ Women's Cancer, Institute for Women's Health, University College London, Maple House 1st Floor, 149 Tottenham Court Road, London W1T 7DN, UK; ${ }^{91}$ Center for Cancer Prevention and Translational Genomics, Samuel Oschin Cancer Institute, Spielberg Building, 8725 Alden Dr., Los Angeles, CA 90048, USA; ${ }^{92}$ School of Women's and Children's Health, University of New South Wales, Level 1, Women's Health Institute, Royal Hospital for Women, Barker Street, Randwick, NSW 2031, Australia; ${ }^{93}$ The Kinghorn Cancer Centre, Garvan Institute of Medical Research, 384 Victoria Street, Darlinghurst, NSW 2010, Australia and ${ }^{94}$ Department of Pathology and Laboratory Diagnostics, The Maria Sklodowska-Curie Memorial Cancer Center and Institute of Oncology, Roentgena 5, 02-781 Warsaw, Poland 